\title{
TU/e EmonOWEN

\section{Modeling optical behavior of birefringent biological tissues for evaluation of quantitative polarized light microscopy}

\section{Citation for published version (APA):}

Turnhout, van, M. C., Kranenbarg, S., \& Leeuwen, van, J. L. (2009). Modeling optical behavior of birefringent biological tissues for evaluation of quantitative polarized light microscopy. Journal of Biomedical Optics, 14(5), 054018-1/11. [054018]. https://doi.org/10.1117/1.3241986

DOI:

10.1117/1.3241986

Document status and date:

Published: 01/01/2009

\section{Document Version:}

Publisher's PDF, also known as Version of Record (includes final page, issue and volume numbers)

\section{Please check the document version of this publication:}

- A submitted manuscript is the version of the article upon submission and before peer-review. There can be important differences between the submitted version and the official published version of record. People interested in the research are advised to contact the author for the final version of the publication, or visit the $\mathrm{DOI}$ to the publisher's website.

- The final author version and the galley proof are versions of the publication after peer review.

- The final published version features the final layout of the paper including the volume, issue and page numbers.

Link to publication

\section{General rights}

Copyright and moral rights for the publications made accessible in the public portal are retained by the authors and/or other copyright owners and it is a condition of accessing publications that users recognise and abide by the legal requirements associated with these rights.

- Users may download and print one copy of any publication from the public portal for the purpose of private study or research.

- You may not further distribute the material or use it for any profit-making activity or commercial gain

- You may freely distribute the URL identifying the publication in the public portal.

If the publication is distributed under the terms of Article 25fa of the Dutch Copyright Act, indicated by the "Taverne" license above, please follow below link for the End User Agreement:

www.tue.nl/taverne

Take down policy

If you believe that this document breaches copyright please contact us at:

openaccess@tue.nl

providing details and we will investigate your claim. 


\section{Modeling optical behavior of birefringent biological tissues for evaluation of quantitative polarized light microscopy}

\author{
Mark C. van Turnhout \\ Sander Kranenbarg \\ Johan L. van Leeuwen \\ Wageningen University \\ Department of Animal Sciences \\ Experimental Zoology Group \\ P.O. Box 338 \\ Wageningen, $6700 \mathrm{AH}$ \\ The Netherlands \\ E-mail: mark.vanturnhout@wur.nl
}

\begin{abstract}
Quantitative polarized light microscopy (qPLM) is a popular tool for the investigation of birefringent architectures in biological tissues. Collagen, the most abundant protein in mammals, is such a birefringent material. Interpretation of results of qPLM in terms of collagen network architecture and anisotropy is challenging, because different collagen networks may yield equal qPLM results. We created a model and used the linear optical behavior of collagen to construct a Jones or Mueller matrix for a histological cartilage section in an optical qPLM train. Histological sections of tendon were used to validate the basic assumption of the model. Results show that information on collagen densities is needed for the interpretation of qPLM results in terms of collagen anisotropy. A parameter that is independent of the optical system and that measures collagen fiber anisotropy is introduced, and its physical interpretation is discussed. With our results, we can quantify which part of different qPLM results is due to differences in collagen densities and which part is due to changes in the collagen network. Because collagen fiber orientation and anisotropy are important for tissue function, these results can improve the biological and medical relevance of qPLM results. () 2009 Society of PhotoOptical Instrumentation Engineers. [DOI: 10.1117/1.3241986]
\end{abstract}

Keywords: mathematical modeling; polarized light microscopy (PLM); cartilage; collagen network; collagen density; collagen anisotropy.

Paper 08355RR received Oct. 6, 2008; revised manuscript received Jun. 6, 2009; accepted for publication Jul. 28, 2009; published online Oct. 12, 2009.

\section{Introduction}

Collagen is the most abundant protein in mammals. ${ }^{1}$ The architecture of the collagen fiber network in various tissues is determined by functional demands on the tissue. Helical collagen architectures are found for instance, in cylindrical flexible hydrostats such as the notochord, ${ }^{2,3}$ the epidermis of cylindrical animals, ${ }^{4,5}$ and the annulus fibrosus of intervertebral discs. $^{6,7}$ Helically arranged collagen fibers in chameleon tongues are subject to large deformations and serve to store energy. ${ }^{8,9}$ In contrast, hydrostats that require resistance against bending show an orthogonal collagen architecture. ${ }^{10,11}$

The mechanical environment also plays an important role for the collagen architecture in, e.g., cardiovascular structures, ${ }^{12,13}$ the intestine, ${ }^{14}$ and articular cartilage. ${ }^{15-17}$ Functional demands can also be optical. The cornea and sclera both need strength to resist the inner pressure of the eye. Yet we find different collagen architectures in these tissues, because the cornea needs to be transparent and the sclera needs to be totally opaque. ${ }^{18,19}$

Polarized light microscopy (PLM) is a popular technique to evaluate collagen architectures in a variety of biological

Address all correspondence to: Mark C. van Turnhout, Wageningen University, Experimental Zoology Group, Department of Animal Sciences, P.O. Box 338 , $6700 \mathrm{AH}$, Wageningen, The Netherlands. Tel: +31 (0)317 483509; Fax: +31 (0)317 483955; E-mail: mark.vanturnhout@wur.nl tissues due to the collagen's (intrinsic) birefringent properties, see, e.g., Refs. 13, 14, and 20-25. PLM has further proven itself to be useful for the investigation of, e.g., retinal nerve fiber layers, ${ }^{26}$ the zona pellucida, ${ }^{27,28}$ meiotic spindles, ${ }^{29}$ and microtubules. $^{30,31}$ In relation to articular cartilage (AC), qPLM has been called "the gold standard of histology.", 24,32

In a pioneering PLM study on $\mathrm{AC}$, Benninghoff ${ }^{33}$ looked at $\mathrm{AC}$ that was positioned between two crossed polarizers. In this setup, anisotropic birefringent architectures appear bright when positioned at $\pm 45 \mathrm{deg}$ with the axis of the polarizers and go extinct when the sample is rotated to \pm 0 deg. Isotropic birefringent architectures and nonbirefringent architectures appear dark when positioned between two crossed polarizers, irrespective of the rotation angle.

With the articular surface of the histological section at $\pm 45 \mathrm{deg}$ with the axis of the polarizers, the superficial zone and deep zone of the AC appear bright and go extinct when the sample is rotated. These zones are separated by a dark transitional zone, which shows little variation in light intensity when the sample is rotated. In these and some additional observations, Benninghoff found an arcade-like architecture, with fibers arranged perpendicularly to the tidemark in the deep zone; fibers that bend away in the transitional zone, forming a more or less random architecture; and fibers aligned

1083-3668/2009/14(5)/054018/11/\$25.00 ㄷ 2009 SPIE 
parallel with the surface in a thin zone at the articular surface. Benninghoff already noted that the arcade architecture serves as a model for the predominant fiber orientation only, a point that has particularly been stressed by scanning electron microscope (SEM) studies, e.g., Refs. 34 and 35. But where Benninghoff had to rely on qualitative measurements, advances in PLM now allow a quantitative analysis of PLM (qPLM) results. $^{36-39}$

Birefringence is an intrinsic optical material property of collagen fibers. With qPLM, two parameters that are related to the birefringent architecture can be determined. We will use retardance to indicate measured extrinsic optical retardations. ${ }^{32,39,40}$ This property is sometimes also called optical path difference, ${ }^{41-43}$ birefringence intensity, ${ }^{17,44}$ or rotation independent birefringence. ${ }^{45}$ From retardances measured with different states of polarized light, we can calculate the azimuth. This is the measured predominant orientation of the birefringent structures in the plane of imaging.

Långsö et al. ${ }^{46}$ write that "further investigations on the role of quantitative PLM in collagen fibril network studies are clearly warranted," and Oldenbourg ${ }^{47}$ remarks that "The art and science of relating measured retardance and azimuth to structural information [on the molecular level] of the specimen is only in its infancy." Rieppo et al. ${ }^{37}$ remark that retardance alone cannot fully characterize the collagen architecture because it is influenced by both collagen density and the structural anisotropy of the fiber architecture.

To the best of our knowledge, a mathematical model that predicts qPLM results for given collagen structures has not been reported in the literature. Such a model is useful to investigate the merits and limitations of qPLM measurements for certain applications. Because collagen behaves as a linear retarder, ${ }^{23,25,26,30,48-51}$ we can construct the optical effect of a given sample with the same mathematical framework that describes the effect of an optical train.

In the present paper, we will use the linear optical behavior of collagen to construct a Jones or Mueller matrix for a histological cartilage section in an optical qPLM train. We show how the intrinsic optical properties of a birefringent fiber network influence qPLM results and show how knowledge of fiber densities can help to interpret these results. We will use $\mathrm{AC}$ as our example tissue for the interpretation of qPLM results and tendon to validate the basic assumption of the model.

\section{Methods}

We simulated the effect of the LC-PolScope system for quantitative $\mathrm{PLM}^{47,52}$ for given collagen architectures. We used Jones calculus ${ }^{53}$ to numerically simulate the intensity of the light on the camera as a result of the optical train and the sample therein; see Sec. 2.1. This was done with different polarization states of the incident light on the sample, which resulted in different (simulated) light intensities. Next, we analyzed these intensities as implemented in the LC-PolScope system $^{54}$ to evaluate the effects of different collagen architectures.

The angles we used to define the collagen architectures will be denoted by $\varphi$, and the birefringence of a single fiber with unit density is $\delta$. Simulated or measured retardance will be $\Delta$, and simulated or measured predominant fiber orientation will be $\phi$.

We considered light that passes a sample of unit thickness with fibers of unit length and equal intrinsic birefringence. We were interested in the height-dependent collagen architecture from tidemark to articular surface, or $0 \leqslant h \leqslant 1$, with $h$ the normalized dimensionless cartilage height. The procedures described in the following were implemented in MATLAB (version 7.6 R2008a, The MathWorks, Inc., 1984-2005) and Octave (version 3.0.1, www.octave.org, 2009).

\subsection{Simulation of Optical Train and Sample}

We can simulate the optical train either with Jones calculus or with Mueller matrices and Stokes vectors. Solutions of the two methods are equal in the case of fully polarized light. ${ }^{55,56}$ We present the Jones calculus in this section and collect the corresponding Mueller matrices in the appendix (Sec. 6). For each optical element in an optical train, Jones ${ }^{53}$ defines two matrices. The orientation of the element is described by a rotation matrix $R$ that depends on orientation angle $\theta$ as

$$
R(\theta)=\left[\begin{array}{cc}
\cos \theta & -\sin \theta \\
\sin \theta & \cos \theta
\end{array}\right] .
$$

The orientation-independent effect is described by a diagonal matrix $N$ :

$$
N=\left[\begin{array}{cc}
N_{x} & 0 \\
0 & N_{y}
\end{array}\right] .
$$

Matrices $N$ for an ideal polarizer $N_{P}$ and an ideal retarder $N_{R}$ with retardance $\varepsilon$ are given by

$$
N_{P}=\left[\begin{array}{ll}
1 & 0 \\
0 & 0
\end{array}\right], \quad N_{R}(\varepsilon)=\left[\begin{array}{cc}
\mathrm{e}^{-\mathrm{i} \varepsilon / 2} & 0 \\
0 & \mathrm{e}^{\mathrm{i} \varepsilon / 2}
\end{array}\right] .
$$

The total effect of the element can then be written as

$$
M(\theta, \varepsilon)=R(\theta) N(\varepsilon) R(-\theta) .
$$

In the polarization microscope we investigate, light passes a linear polarizer $P$, a quarter wave plate $Q$, the sample $S$, two liquid crystals or variable retardance plates $L_{b}$ and $L_{a}$, and last a linear analyzer $A$ (see Fig. 1). With $J_{0}$ the vector of the (unpolarized) incident light, the description of the light that reaches the camera $J$ is given by

$$
J=A \cdot L_{a} \cdot L_{b} \cdot S \cdot Q \cdot P \cdot J_{0},
$$

with the characteristics (rotation, retardation) as gathered in Table 1 . Note that the liquid crystals $L_{a}$ and $L_{b}$ apply a retardance of $\alpha$ and $\beta$, respectively, values that are set by the analysis software.

The sample is composed of $N_{f}$ collagen fibers that are modeled as ideal linear retarders. To find the matrix $S$ for the sample, we therefore calculate for $N_{f}$ fiber directions: 


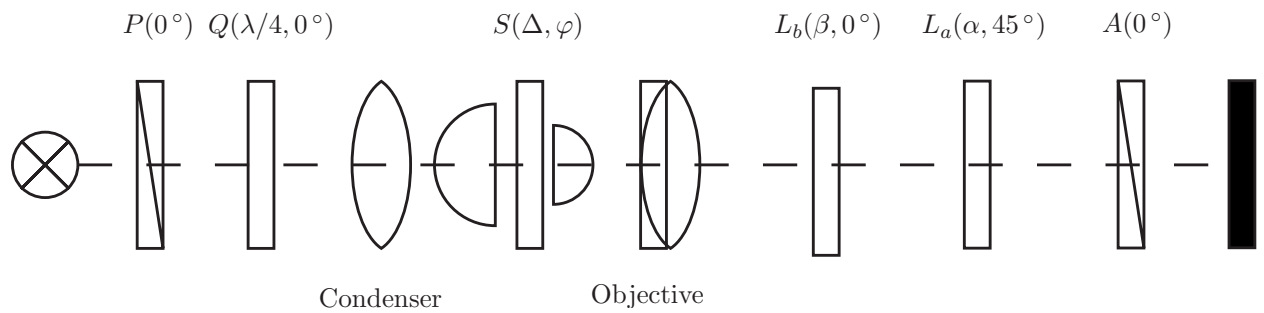

Fig. 1 Optical train of the polarization microscope, adapted from Shribak and Oldenbourg (Ref. 54, figure 1b). Light passes a linear polarizer $P$, a quarter wave plate $Q$, the sample $S$, two liquid crystals or variable retardance plates $L_{b}$ and $L_{a}$, and last a linear analyzer $A$.

$$
S=\prod_{n=1}^{N_{f}} R[\varphi(n)]\left\{\begin{array}{cc}
\exp \left[-\mathrm{i} \frac{\eta(n) \delta}{2}\right] & 0 \\
0 & \exp \left[\frac{\eta(n) \delta}{2}\right]
\end{array}\right\} R[-\varphi(n)],
$$

with $\varphi(n)$ the angle, $\eta(n)$ the (relative) collagen density in the $n$ 'th direction, and $\delta$ the birefringence of a single fiber with unit density.

In our simulations and analysis, we use the five-frame algorithm as described by Shribak and Oldenbourg. ${ }^{54}$ Thus, we simulate five intensities with five settings for the liquid crystals $(\alpha$ and $\beta)$. The analytical solution for the intensity in a pixel in this (ideal) system is

$$
\begin{aligned}
I= & \frac{I_{0}}{4}[1+\cos \alpha \sin \Delta \sin 2 \phi-\sin \alpha(\sin \beta \sin \Delta \cos 2 \phi \\
& -\cos \beta \cos \Delta)],
\end{aligned}
$$

with $I_{0}$ the intensity of the incident unpolarized light, $\phi$ the effective azimuth, and $\Delta$ the effective retardance of the sample. With the five intensities, we can eliminate the unknowns and find analytical expressions for $\phi$ and $\Delta$ as a function of the intensities (Ref. 54, Eq. 20).

\subsection{Collagen Fiber Network}

We used two collagen networks for the simulations: a Benninghoff network and a gothic network. The networks were assumed uniform over the width (and thickness) of the sample, so we could use 1-D patterns for our examples. The first collagen fiber network was inspired by the Benninghoff model and modeled with an arcade. At the tidemark, fibers are aligned perpendicularly to the tidemark, and at the articular

Table 1 Characteristics of the standard elements in the optical train.

\begin{tabular}{lll}
\hline Element & Type & Variables \\
\hline$P$ & Polarizer & $\theta=0$ \\
$Q$ & Retarder & $\theta=0, \varepsilon=\frac{\pi}{2}$ \\
$L_{b}$ & Retarder & $\theta=0, \varepsilon=\beta$ \\
$L_{a}$ & Retarder & $\theta=\frac{\pi}{4}, \varepsilon=\alpha$ \\
$A$ & Polarizer & $\theta=0$ \\
\hline
\end{tabular}

surface, fibers are aligned parallel with the surface. We described this Benninghoff network with two height-dependent angles $\varphi$ :

$$
\varphi_{1,2}=90 \mathrm{deg} \pm \operatorname{atan}\left(\frac{h}{a \sqrt{1-h^{2}}}\right),
$$

with $a=1.2$. For the second collagen fiber network, we also let fibers start perpendicular to the tidemark and use a linear angle definition for $\varphi_{1,2}$ :

$$
\varphi_{1,2}=90 \mathrm{deg} \pm b h,
$$

with $b=58.44 \mathrm{deg}$. Fiber orientations for these 2-D networks are shown in Fig. 2.

For 3-D networks, we need to project the collagen fiber onto the plane of imaging. A fiber that is defined in the plane
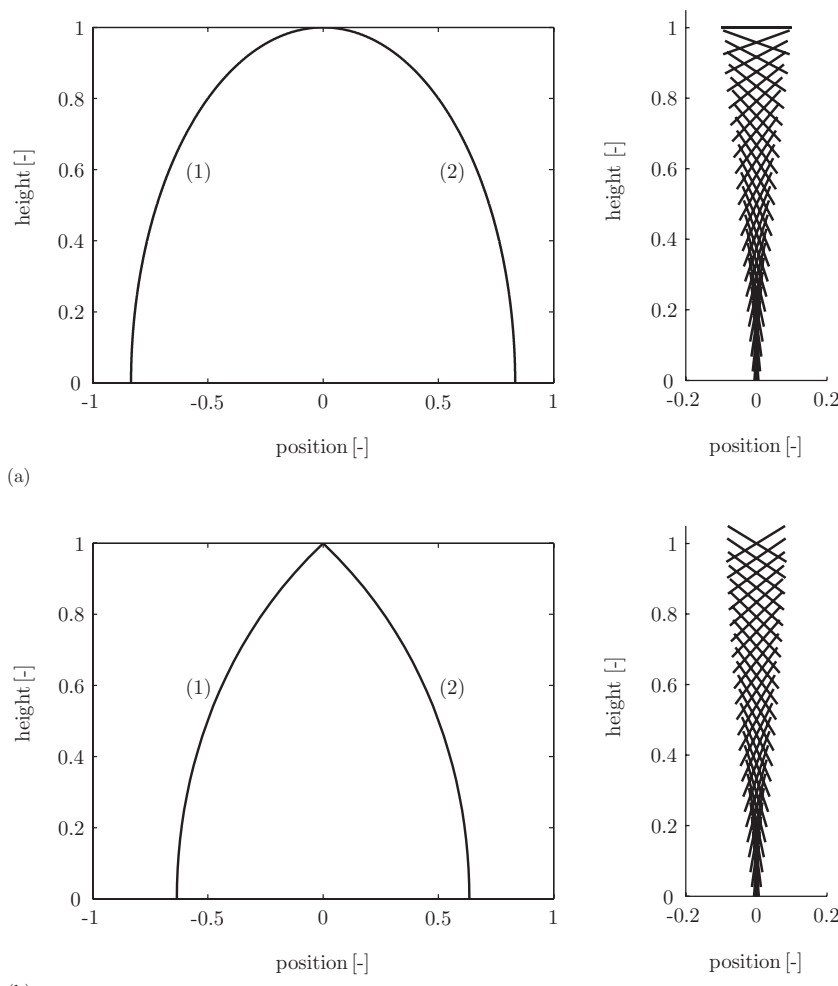

Fig. 2 Collagen networks used in the simulations. Left: Arcades described by two fibers $(1,2)$ as a function of normalized height. Right: Orientations for the two fibers in a series of points over the normalized height. (a) Benninghoff network; (b) gothic network. 


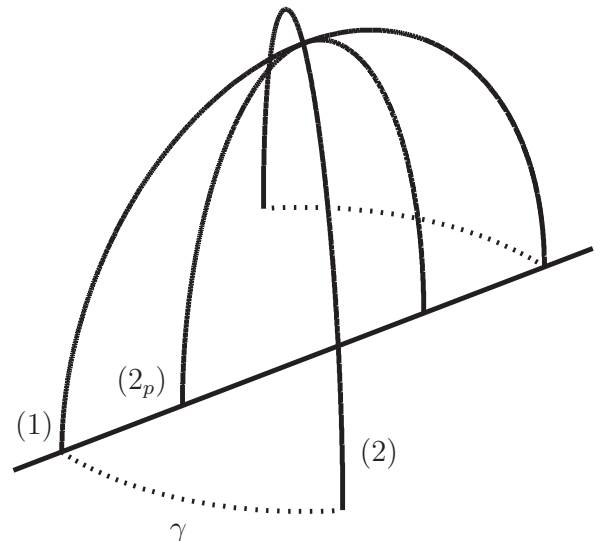

Fig. 3 For 3-D networks, we project fibers onto the plane of imaging. The arcade that is defined in the plane of imaging (1) is rotated over angle $\gamma$ out of the plane of imaging to give arcade (2). To analyze this arcade, we project it onto the plane of imaging, arcade $(2 p)$.

of imaging with angle $\varphi$ and density $\rho$ and rotated out of this plane around an axis in the direction of the height of the sample will have projected angles

$$
\varphi_{p}=\operatorname{atan}\left(\frac{\tan \varphi}{\cos \gamma}\right),
$$

and projected density

$$
\rho_{p}=\rho\left(\cos ^{2} \varphi \sin ^{2} \gamma+\sin ^{2} \varphi\right)^{1 / 2},
$$

with $\gamma$ the angle of rotation. This illustrated in Fig. 3 .

We used two Benninghoff arcades to model 3-D networks for the simulations. In the simulations, we first used one Benninghoff arcade in the plane of imaging (as in the 2-D simulations) and a second Benninghoff arcade that is rotated out of the plane of imaging over three angles: $0 \mathrm{deg}, 45 \mathrm{deg}$, and $90 \mathrm{deg}$. Second, we used a fixed network with two Benninghoff arcades with an angle of 45 deg between them and rotated the plane of imaging over three angles: $0 \mathrm{deg}, 45 \mathrm{deg}$, and $90 \mathrm{deg}$. When the plane of imaging is at $0 \mathrm{deg}$, one arcade is rotated over $22.5 \mathrm{deg}$, and one arcade is rotated over -22.5 deg compared to the plane of imaging.

The Benninghoff network represents predominant fiber orientations only. Therefore, we introduced a second fiber network to model a random (macroscopically isotropic) fiber network. This is a "zero retardance background" network that by definition does not influence the analyzed azimuth values. Such a zero retardance network can be modeled with only two fibers in the plane of imaging that are perpendicular to each other. In our analysis, we chose to define two angles for this network: $45 \mathrm{deg}$ and $135 \mathrm{deg}$. A profile for the heightdependent distribution of collagen density in $\mathrm{AC}$ was taken from Venn and Maroudas ${ }^{57}$ and scaled to have a maximum of unity. This profile is described by

$$
\rho_{t}(h)=1.37 h^{2}-1.49 h+1,
$$

and is shown in Fig. 4.

Collagen density [Eq. (12)] is divided over two networks, and within the networks over individual fibers. With $V$ the

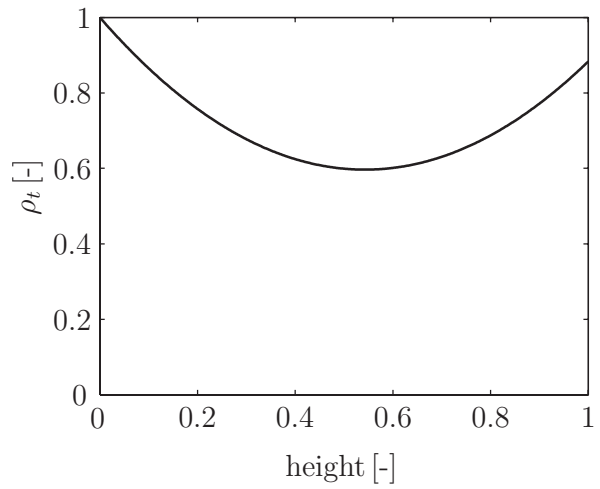

Fig. 4 Profile for collagen density as function of dimensionless height. Adopted from Venn and Maroudas ${ }^{57}$ and scaled to have a maximum of one.

fraction of the total projected collagen density $\rho_{p}$ that is represented by the Benninghoff network, we can write for individual fibers:

$$
\begin{gathered}
\eta_{1}(h)=\frac{\rho_{p}(h)}{n_{f_{1}}} V(h), \\
\eta_{2}(h)=\frac{\rho_{p}(h)}{n_{f_{2}}}[1-V(h)],
\end{gathered}
$$

where $\eta_{1}$ represents relative collagen density for $n_{f_{1}}$ individual orientations in the Benninghoff network, and $\eta_{2}$ represents relative collagen density for $n_{f_{2}}$ individual orientations in the zero retardance network.

\subsection{Experimental Validation}

To validate the basic assumption in the modeling of the sample, we performed experiments with sheep tendon. We tested in 2-D whether we could predict retardance and azimuth results for a known simple network that we constructed with tendon. This experiment tests the linear optical behavior of collagen fibers.

Sheep tendon (superficial sesamoidean ligament and deep flexor tendon) was isolated and cut to a length of $5 \mathrm{~cm}$. Isolated tendon was stretched on a small board with screws at $0.5 \mathrm{~cm}$ of the tendon endings. The board with stretched tendon was fixated at $4{ }^{\circ} \mathrm{C}$ in formalin (4\% in PBS overnight followed by $1 \%$ in PBS overnight), washed four times in PBS and infiltrated with sucrose (20\% in PBS) overnight, and snap frozen in $n$-pentane. Frozen tendon was removed from the board and stored at $-20^{\circ} \mathrm{C}$. Transverse sections (thickness $6 \mu \mathrm{m}$ ) were cut on a cryostat, put on a microscopy glass with a cover glass, and mounted with aquamount.

Mounted tendon sections were analyzed with the LCPolScope system for qPLM. ${ }^{47,52}$ Images were obtained with a Zeiss Axiovert $200 \mathrm{M}$ microscope at a $20 \times / 1 \times$ magnification, equipped with a Q-imaging monochrome HR Retiga EX 1350 camera. Recorded intensity images had a resolution of $0.62 \times 0.62 \mu \mathrm{m}^{2} /$ pixel and were stored in 8 -bit TIFF format. We used the five-frame setting with background correction as described by Ref. 54 with a swing of $0.1[-]$. The recorded images were analyzed for predominant collagen fibril orienta- 

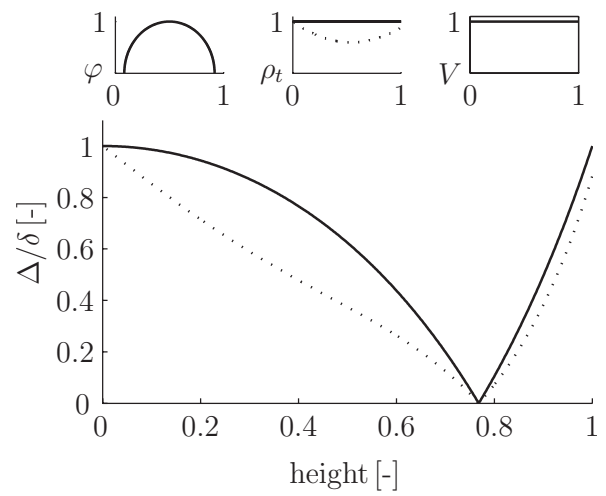

Fig. 5 Retardance patterns (main panel) for two simulations with the Benninghoff network (top-left panel), with collagen density (topmiddle panel) set to unity (solid) or adapted from Venn and Maroudas $^{57}$ (dashed), and $V$ (top-right panel) set to unity. Horizontal axes in the panels represent cartilage height as in the main panel.

tion and tissue retardance with custom written scripts implemented in MATLAB (version 7.6 R2008a, The MathWorks, Inc., 1984-2005).

Two glasses with tendon were placed on top of each other, and three image stacks were recorded: one with the lower tendon in focus, one with the upper tendon in focus, and one with focus between the two tendons. Images were analyzed for average retardance and azimuth in a $50 \times 50 \mathrm{pixel}^{2}$ box in three positions: one for each tendon where there is no overlap with the other tendon, and one where the tendons overlap. We used the mathematical model with one point and two fibers to model the retardance $\Delta$ and azimuth $\phi$ for the position where the tendons overlap. Each tendon was represented by a collagen fiber with unit density and birefringence $\delta$ and azimuth $\varphi$ as measured in the nonoverlapping position. Predictions of the model were compared to the measured results at the position of overlap.

\section{Results}

\subsection{2-D Retardance}

Figure 5 displays the results for two analyses with the Benninghoff network only $(V=1)$ and two collagen density profiles. Retardance is zero for both analyses at the point where the two fibers that form the arcade are perpendicular to each other. This happens at $h=\left[a^{2} /\left(1+a^{2}\right)\right]^{1 / 2}$ in our example, i.e., with $a=1.2$ at $h=0.77$. With collagen density set to unity, we find the maximum possible retardance $\Delta / \delta=1$ at the points where the two fibers that form the arcade are parallel to each other: in our example, at $h=0$ and $h=1$. With both collagen density and $V$ set to unity, the retardance pattern represents the effects of collagen orientation only. With the nonconstant collagen density profile, we find that maximum possible retardance $\Delta / \delta$ no longer equals 1 , but that $\Delta / \delta \leqslant \rho_{t}$ because $\Delta_{\rho=\rho_{t}}=\rho_{t} \Delta_{\rho=1}$. Retardance in this pattern therefore represents the effects of both collagen orientation and collagen density. Note that total collagen density is divided over two fibers and that therefore each fiber has a relative density of $\rho_{t} / 2$.

The effect of parameter $V$ is illustrated in Fig. 6. This shows the retardance pattern for the gothic network with collagen density and $V$ set to unity, and a retardance pattern for
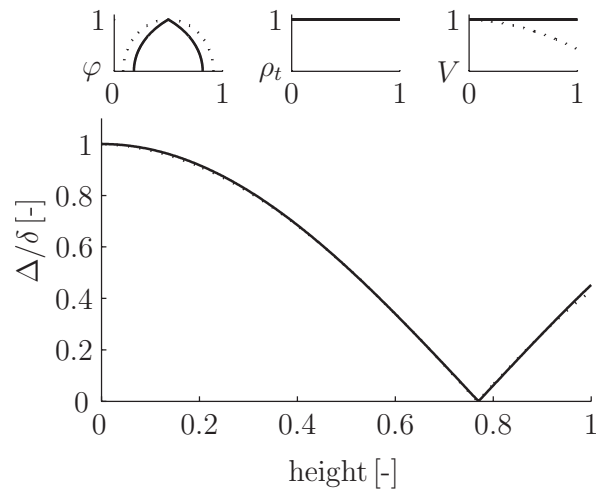

Fig. 6 Two collagen networks with equal retardance results. Solid: Gothic network (top-left panel), with collagen density (top-middle panel) and $V$ (top-right panel) set to unity. Dashed: Benninghoff network (top-left panel), with collagen density (top-middle panel) set to unity and values for $V$ (top-right panel) that result in the retardance pattern we observed for the gothic network (solid). Horizontal axes in the panels represent cartilage height as in the main panel.

the Benninghoff network with collagen density set to unity and $V$ a function of height. We note two things: first, that the effect of $V$ on the retardance pattern is equal to that of $\rho_{t}$ : $\Delta_{V=V(h)}=V(h) \Delta_{V=1}$, and second, that different collagen networks can result in equal retardance patterns.

\subsection{2-D Azimuth}

Figure 7 shows the azimuth results for the 2-D simulations. Because of our choices for the definitions of the two networks, these results are the same for all 2-D simulations. The figure further shows that analyzed azimuth does not change when total collagen density is adopted, or when more or less collagen is associated with the zero retardance background network (decreasing $V$ ).

\subsection{3-D Simulations}

Figure 8 shows the results for simulations with one Benninghoff arcade in the plane of imaging (as in the 2-D simulations) and one arcade rotated out of the plane of imaging over three

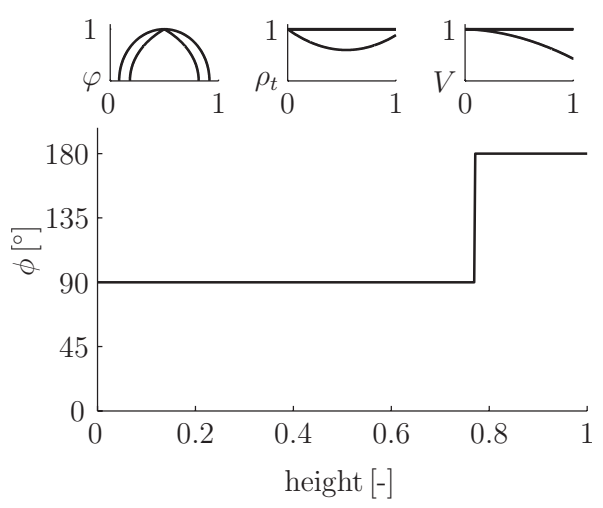

Fig. 7 Azimuth results for the 2-D simulations. Azimuth (main panel) is 90 deg for $h<0.77$ and 180 deg elsewhere for each combination of Benninghoff or gothic network (top-left panel), with constant or height-dependent collagen density (top-middle panel) and with constant or height-dependent $V$ (top-right panel). Horizontal axes in the panels represent cartilage height as in the main panel. 

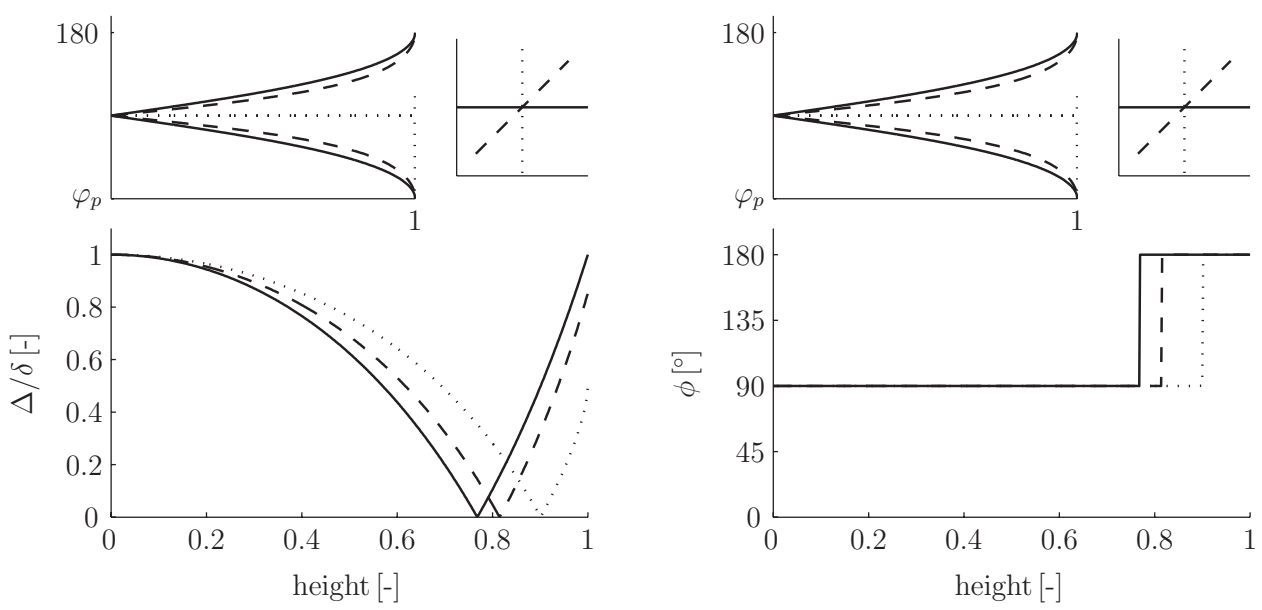

Fig. 8 Results for three 3-D networks with two Benninghoff arcades. Left: Retardance. Right: Azimuth. One arcade (solid) is in the plane of imaging; the second arcade is rotated out the plane of imaging over 0 deg (solid), 45 deg (dashed), and 90 deg (dotted). Top-left panels: Projected fibril angles for the rotated fibers $\left(0 \leqslant h \leqslant 1,0 \mathrm{deg} \leqslant \varphi_{p} \leqslant 180 \mathrm{deg}\right)$. Top-right panels: Top view of the networks, where the solid line represents one arcade in the plane of imaging.

angles: $0 \mathrm{deg}, 45 \mathrm{deg}$, and $90 \mathrm{deg}$. With both arcades in the plane of imaging, the results are equal to those from the 2-D simulations with collagen density and $V$ set to unity (see Figs. 5 and 7). When an arcade is rotated out of the plane of imaging, we see that the point where the (projected) network behaves as isotropic-i.e., where retardance is zero and where analysed azimuth changes from $90 \mathrm{deg}$ to $180 \mathrm{deg}$ - shifts toward $h=1$. Retardance near $h=1$ becomes smaller as the second fiber is rotated more out of the plane of imaging. Note that total collagen density is now divided over four fibers and that therefore each fiber has a relative density of $\rho_{t} / 4$.

In Fig. 9, we look at a single 3-D network with three different orientations of the plane of imaging: $0 \mathrm{deg}, 45 \mathrm{deg}$, and $90 \mathrm{deg}$. When this network is viewed from the top, there is a predominant direction, and the plane of imaging at $0 \mathrm{deg}$ is in the direction of this predominant direction. When the plane of imaging is rotated away from the predominant direction $(0 \mathrm{deg})$, we see that the point where the (projected) network behaves as isotropic-i.e., where retardance is zero and where analyzed azimuth changes from $90 \mathrm{deg}$ to $180 \mathrm{deg}-$ shifts toward $h=1$. Retardance near $h=1$ becomes smaller as the plane of imaging is rotated farther compared to the predominant direction $(0 \mathrm{deg})$.

\subsection{Experimental Validation}

For the experiments, results of three images with different focus were compared for each combination of two tendon sections. We checked whether the exact location of the 50 $\times 50$ pixel $^{2}$ box or the size of the box influenced the results. There were no notable differences between choices of focus, boxes that were moved over the tendons, or size of the box. We decided to present the data from the image that has the focus between the two sections.

Figure 10 shows a representative image with focus between two tendon sections and the boxes that were used for the analysis. Results for four combinations with two sections are collected in Table 2. The predictions for the azimuth of the combinations of tendons are excellent. The predictions for the retardance of the combinations of tendons differ approximately $10 \%$ from the measurements.

\section{Discussion}

It is well known that collagen fiber anisotropy and collagen density influence qPLM results, e.g., Refs. 17, 24, 41, 44, and 46. In the 2-D simulations in the theoretical part of this paper, we wrote collagen orientation as the sum of two networks: one representing the predominant orientations, and one representing an isotropic network that does not influence the azimuth results and has a retardance of zero. Total collagen density $\rho_{t}$ was divided over these two networks: $\rho_{t} V$ for the predominant orientations, and $\rho_{t}(1-V)$ for the isotropic network. Results are determined only by collagen in the predominant network, with relative density $\rho_{t} V$. This explains

Table 2 Results for the tendon experiments. Four combinations of two tendons each were analyzed with gPLM. Results for single tendons (columns 2 and 3 ) were used to predict the experimental outcome of the combination of the two tendons. The experimental outome is given in column 4; the model prediction is given in column 5 . Results for azimuth are on top in the table; results for retardance are at the bottom in the table.

Tendon 1 Tendon 2 Tendon 1 and 2 Prediction

\begin{tabular}{lcccc}
\hline Azimuth (deg) & & & & \\
Combination 1 & 156 & 73 & 152 & 153 \\
Combination 2 & 161 & 86 & 158 & 157 \\
Combination 3 & 81 & 1.2 & 77 & 73 \\
Combination 4 & 178 & 118 & 122 & 124 \\
\hline Retardance (nm) & & & & \\
Combination 1 & 124 & 30 & 100 & 95 \\
Combination 2 & 129 & 23 & 99 & 110 \\
Combination 3 & 122 & 54 & 83 & 72 \\
Combination 4 & 54 & 118 & 103 & 95 \\
\hline
\end{tabular}



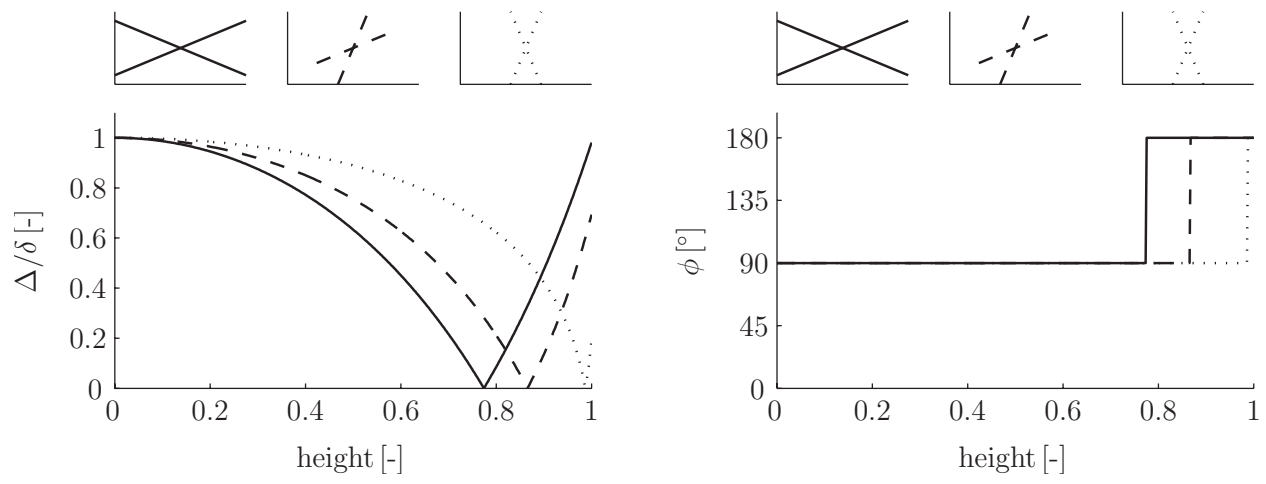

Fig. 9 Results for a 3-D network with two Benninghoff arcades. Left: Retardance. Right: Azimuth. Top panels show a top view of the two arcades in the networks, where the plane of imaging is represented by the horizontal.

why the effect of decreasing total collagen density $\rho_{t}$ with a certain factor equals the effect of decreasing the (relative) amount of collagen in the predominant network $V$. Interpretation between $\rho_{t}$ and $V$ differs, however. Total collagen density $\rho_{t}$ is a parameter with physical meaning that can be measured outside qPLM. $V$ is the fraction of total collagen that contributes to qPLM results: when collagen is present but fully incorporated in an isotropic network, we use physical values for $\rho_{t}$ with $V=0$ and arrive at the correct results.

The 2-D simulations further stress that different collagen networks can give equal qPLM results. Reconstruction of the collagen network from qPLM results is therefore not possible. Without information about collagen densities, the best we can do is to interpret azimuth results as a predominant orientation $\phi(h)$ and the retardance $\Delta(h)$ as a measure for the amount of collagen associated with this predominant angle. When collagen densities are known, we can do better. The retardance $\Delta(h)$ associated with a certain angle divided by the total amount of collagen $\rho_{t}(h)$ can be interpreted as a measure of network anisotropy: $\Delta / \rho_{t}=V$. When $V=1$, we measure maximum possible retardance for the given amount of collagen, which means that anisotropy is maximum and all fibers are oriented parallel. For $V=0$ (when $\Delta=0$ ), there is no collagen associated with the measured predominant direction, which means that we are looking at isotropic fiber orientations.

We illustrate this with results on cartilage retardance and collagen densities presented by Rieppo et al. for immature pigs (4 months old) and mature pigs (21 months old) (Ref. 58, figure 6). Collagen content increases from the immature sections to the mature sections. If the organization of the collagen network in the mature section is equal to that of the collagen network in the immature section, retardance will scale directly with collagen content. If a collagen network is added that is more anisotropic than the immature network, retardance will increase more. If a collagen network is added that is less anisotropic than the immature network, retardance will increase less. At the articular surface, collagen content increases $40 \%$, from 0.13 [-] for the immature pigs to 0.22 [-] for the mature pigs, and retardance increases $20 \%$, from $0.38 \cdot 10^{-3}[-]$ for the immature pigs to $0.48 \cdot 10^{-3}[-]$ for the mature pigs. Thus, the mature network is less anisotropic than the immature network. In terms of the model, the fraction of collagen that is associated with the predominant orientation, $V$, is smaller. At a distance of $800 \mu \mathrm{m}$ from the articular surface, collagen content increases $45 \%$, from $0.2[-]$ for the immature pigs to 0.37 [-] for the mature pigs, and retardance increases $70 \%$, from $0.6 \cdot 10^{-3}[-]$ for the immature pigs to $2 \cdot 10^{-3}[-]$ for the mature pigs. Thus, the mature network is more anisotropic than the immature network. In terms of the model, the fraction of collagen that is associated with the predominant orientation, $V$, is larger.

Figure 11 shows $\bar{v}=1000 \cdot($ retardance $) /($ collagen content) for the data of Rieppo et al. ${ }^{58}$ as a function of distance from the articular surface. This $\bar{v}$ differs from $V$ by a scaling factor: $\bar{v}=\kappa V$. In the theoretical part of the study, we retardance $[\mathrm{nm}]$

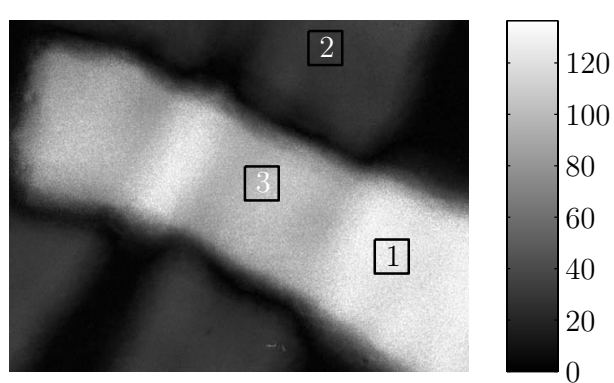

azimuth $\left[^{\circ}\right.$

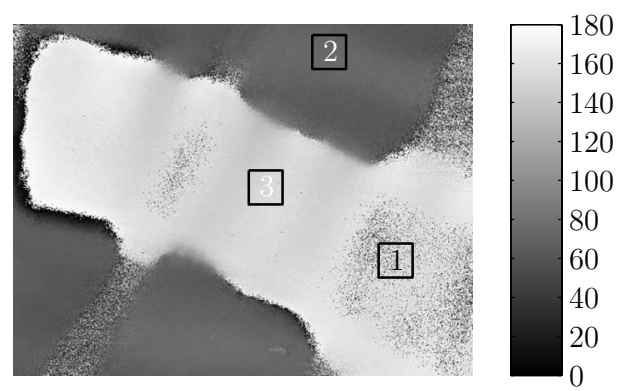

Fig. 10 Example of qPLM results with two tendons on top of each other. Average retardance (left) and azimuth (right) for the squares labeled 1 and 2 (single tendon only) were used to predict the average results in the square labeled 3. Predicted results were compared with the measurement in square 3 . Boxes measure $50 \times 50 \mathrm{pixel}^{2}=31 \times 31 \mu \mathrm{m}^{2} /$ pixel. 


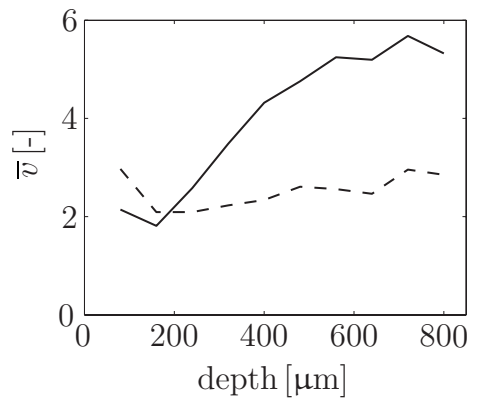

Fig. 11 Measure for the fraction collagen associated with predominant orientation as a function of distance from the articular surface. Calculated from results presented by Rieppo et al. (Ref. 58, figure 6). Solid: Results for a 21-month-old pig. Dashed: Results for a 4-monthold pig.

knew the birefringence per amount of collagen: $\delta=\pi / 180$ per collagen fiber with unit density. We already scaled the retardance patterns with $\delta$ in Sec. 3. The factor $\kappa$ represents the practical version of $\delta$ : birefringence per gram of fully anisotropic collagen, for instance. Because such information is not yet available, we cannot directly interpret $\bar{v}$ as a fraction of the collagen. We can however, compare values of $\bar{v}$ and say that at the articular surface, the fraction of collagen associated with the predominant orientation decreases with $40 \%$ from $\bar{v}=3$ for the immature pigs to $\bar{v}=2.1$ for the mature pigs. At a distance of $800 \mu \mathrm{m}$ from the articular surface, the fraction of collagen associated with the predominant orientation increases with $46 \%$ from $\bar{v}=2.9$ for the immature pigs to $\bar{v}$ $=5.3$ for the mature pigs. An increase of collagen network anisotropy near the tidemark from immature to mature cartilage is in line with SEM results on equine AC. ${ }^{35}$

In another study, Rieppo et al. proposed three parameters as a measure for fiber parallelism. ${ }^{37}$ Their microscope uses crossed polarizers between which the sample is rotated. The minimum light intensity that is observed when the sample is rotated is used for each of the three parallelism parameters. We cannot measure this minimum light intensity with our microscope and therefore cannot use the methods proposed by Rieppo et al. to present information on fiber parallelism. Calculation of $\bar{v}$ is independent of the microscope used, but does need information about collagen densities.

Three-dimensional simulations illustrate that qPLM information remains in principle 2-D: only collagen in the plane of imaging contributes to the results. A collagen network that is not invariant to the plane of cutting will give different results depending on the orientation of the cutting plane. This was experimentally shown by Király et al. ${ }^{42}$ Superficial split line patterns indicate the predominant orientation of collagen fibers in the most superficial cartilage layer across the articular surface. Király et al. found that sections cut perpendicular to these split lines showed decreased retardance in the superficial zone compared to sections cut parallel to the superficial split lines. This is illustrated in Fig. 9 with the dotted lines (perpendicular) and solid lines (parallel). Also, rotation of the section away from the parallel orientation shifts the position where the collagen network is isotropic in the plane of imaging toward the articular surface.
It is well known that measured retardance is a function of both collagen anisotropy and collagen density, e.g., Refs. 17, 24, 41, 44, and 46. Retardance measurements can explicitly be used to indicate the amount of birefringent material, e.g., Refs. 30, 39, and 59. The linear relationship between density of birefringent material and tissue retardance has been described for a variety of tissues, ${ }^{23,25,26,30,48-50}$ and the results presented by Bueno and Jaronski ${ }^{51}$ suggest that collagen is indeed a linear retarder. Also, the linear relationship between collagen density and measured retardance is implicitly assumed when observed retardances are scaled with sample thickness ${ }^{39,40,46,60}$ and experimentally observed in the linear relationship between retardance and sample thickness. ${ }^{27,42}$ Our experiments with tendon confirm this: both retardance and azimuth of combinations of different amounts of birefringent material forming different simple networks can be predicted by the model using linear optical behavior for collagen.

When Rieppo et al. ${ }^{37}$ report that within a histological section of AC, collagen density and retardance need not be linearly related with each other, this is due to the influence of fiber anisotropy that varies over the section. This is illustrated, e.g., in Figs. 5 and 6, where collagen density is constant but retardance varies due to collagen network variations. When the thickness of a histological section is increased, measured retardance will increase linearly with thickness only when it is safe to assume that collagen architecture and density are uniform over the thickness of the section. ${ }^{27,42,61}$

Within a sample, relevant differences in collagen density can exist. In our example, adopted from the literature, ${ }^{57}$ the minimum collagen density is approximately $60 \%$ of the maximum. Such profiles can explain why several authors report an increase in retardance toward the tidemark where collagen density is highest. ${ }^{41}$ If we want to evaluate the difference in retardance in terms of collagen network anisotropy, this difference in density should be taken into account. Also comparison of absolute retardances in terms of collagen anisotropy between samples (particularly of different origin: age, species, joint, pathology, etc.) may prove seriously impaired by a lack of knowledge on the collagen density profiles. The example with the data from Rieppo et al. ${ }^{58}$ shows that we need collagen densities to evaluate what part of the changed retardance between the immature and mature pigs is due to the increase in collagen density and what part is due to collagen network remodeling.

Whether collagen density profiles need to be taken into account depends on the application for the qPLM analysis and the desired interpretation of the results. Traditional zone estimation based on retardance ${ }^{15-17,62,63}$ does not need to suffer from collagen density effects, as long as collagen density is relatively constant in the upper half of the tissue and sections are cut parallel to the superficial split lines. This is the case with the density profile shown in this work. Also, we would have to assume a very unrealistic pattern to explain the lack of retardance in the transitional zone by collagen density alone.

In this paper, we have chosen to concentrate on the relation between collagen orientation, anisotropy, and density and qPLM results. Other components of AC tissue have therefore been neglected, although the proteoglycan molecules are known to contribute to the measured retardance, ${ }^{42}$ for instance, through the mechanism of form birefringence. $41,61,64$ We have furthermore assumed that all collagen fibers share 
the same birefringence $\delta$. This is a crude, but for now necessary, modeling step. For instance, collagen fiber diameter influences its birefringence $e^{16,24}$ and is reported to be depth dependent. $^{41,65,66}$ But little or no quantitative information about the possible influence on measured retardance is known, and we therefore decided to keep these simulations simple.

These effects-e.g., form birefringence and depthdependent birefringence - can be incorporated in Eq. (6) as more information becomes available. Furthermore, the relationship between collagen architecture, anisotropy, and density and retardance and azimuth we present here is directly applicable to all forms of qPLM. ${ }^{36-38,45}$

\section{Conclusions}

With the simulations, we attempt a quantification of the actual architecture of the birefringent material-e.g., collagen in AC. We confirm that the problem is undetermined when one looks for a unique collagen architecture that describes certain qPLM results. Because we can only measure the projection of 3-D orientations onto the plane of imaging, qPLM results and their interpretation will always be limited to the 2-D plane of imaging.

Knowledge of collagen densities can greatly facilitate the interpretation of qPLM results in terms of collagen orientation and anisotropy. Correction of retardances for collagen densities will provide a better structural interpretation of qPLM results. By writing the collagen network as the sum of an anisotropic network with a single predominant orientation and an isotropic network, we have arrived at a parameter $\bar{v}=\kappa V$ that has physical meaning and can be seen as a measure for collagen anisotropy. Retardance is a measure for the absolute amount of collagen that can be associated with the predomi-

$$
\begin{aligned}
& J=\left[\begin{array}{cc}
\cos \frac{\varepsilon}{2}+\mathrm{i} \sin \frac{\varepsilon}{2} \cos 2 \theta & \mathrm{i} \sin \frac{\varepsilon}{2} \sin 2 \theta \\
\mathrm{i} \sin \frac{\varepsilon}{2} \sin 2 \theta & \cos \frac{\varepsilon}{2}-\mathrm{i} \sin \frac{\varepsilon}{2} \cos 2 \theta
\end{array}\right], \\
& M=\left[\begin{array}{cccc}
1 & 0 & 0 & 0 \\
0 & \cos ^{2} 2 \theta+\cos \varepsilon \sin ^{2} 2 \theta & (1-\cos \varepsilon) \sin 2 \theta \cos 2 \theta & \sin \varepsilon \sin 2 \theta \\
0 & (1-\cos \varepsilon) \sin 2 \theta \cos 2 \theta & \sin ^{2} 2 \theta+\cos \varepsilon \cos ^{2} 2 \theta & -\sin \varepsilon \cos 2 \theta \\
0 & -\sin \varepsilon \sin 2 \theta & \sin \varepsilon \cos 2 \theta & \cos \varepsilon
\end{array}\right]
\end{aligned}
$$

The Mueller matrix for the sample can be obtained from the Jones matrix by calculation of: ${ }^{67}$

$$
M=A\left(J \otimes J^{c}\right) A^{-1}, \quad A=\left[\begin{array}{cccc}
1 & 0 & 0 & 1 \\
1 & 0 & 0 & -1 \\
0 & 1 & 1 & 0 \\
0 & \mathrm{i} & -\mathrm{i} & 0
\end{array}\right],
$$

with $J^{c}$ the complex conjugate of the Jones matrix $J$. The Mueller matrix can also be calculated directly [in line with Eq. (6)] for $N_{f}$ fibers as

$$
M=\prod_{n=1}^{N_{f}} M_{R[\varphi(n), \eta(n) \delta]},
$$

with $M_{R[\varphi(n), \eta(n) \delta]}$ the ideal retarder [Eq. (18)] at azimuth $\varphi(n)$ and with birefringence $\eta(n) \delta$.

\section{References}

1. P. Fratzl, Ed., Collagen: Structure and Mechanics, Springer, New York (2008).

nant orientation; $V=\Delta / \rho$ measures the fraction of collagen

Research into the relationship between collagen architecture and tissue function on the basis of qPLM results alone is eled with this framework when we learn more about single birefringence and its relation to collagen fiber diameter.

We kindly thank Prof. Rudolf Oldenbourg at the Marine Biothank Marcel Jaklofsky and Appendix: Jones and Mueller Matrices

The Jones matrix $J$ and Mueller matrix $M$ of an ideal polar$J=\left[\begin{array}{cc}\cos ^{2} \theta & \sin \theta \cos \theta \\ \sin \theta \cos \theta & \sin ^{2} \theta\end{array}\right]$,

The Jones matrix $J$ and Mueller matrix $M$ for an ideal retarder with phase shift $\varepsilon$ at an azimuth $\theta$ are: ${ }^{.56}$

2. M. Koehl, D. S. Adams, and R. E. Keller, "Mechanical development of the notochord in Xenopus early tail-bud embryos," in N. Akkaş, Ed., "Biomechanics of active movement and deformation of cells," 
NATO ASI Series H, Cell Biology, vol. 42, pp. 471-485, SpringerVerlag, Berlin (1990).

3. D. S. Adams, R. Keller, and M. Koehl, "The mechanics of notochord elongation, straightening, and stiffening in the embryo of Xenopus laevis," Development 110(1), 115-130 (1990).

4. R. Clark and J. Cowey, "Factors controlling the change of shape of certain nemertean and turbellarian worms," J. Exp. Biol. 35(4), 731$750(1958)$

5. L. Picken, M. Pryor, and M. Swann, "Orientation of fibrils in natural membranes," Nature (London) 159, 434 (1947).

6. N. Y. Ignatieva et al., "IR laser and heat-induced changes in annulus fibrosus collagen structure," Photochem. Photobiol. 83(3), 675-685 (2007).

7. J. Yu, U. Tirlapur, J. Fairbank, P. Handford, S. Roberts, C. P. Winlove, Z. Cui, and J. Urban, "Microfibrils, elastin fibers and collagen fibers in the human intervertebral disc and bovine tail disc," J. Anat. 210(4), 460-471 (2007).

8. J. H. de Groot and J. L. van Leeuwen, "Evidence for an elastic projection mechanism in the chameleon tongue," Proc. Biol. Sci. 271(1540), 761-770 (2004).

9. J. L. van Leeuwen, "Why the chameleon has spiral-shaped muscle fibers in its tongue," Philos. Trans. R. Soc. London, Ser. B 352(1353), 573-589 (1997).

10. D. Kelly, "Axial orthogonal fiber reinforcement in the penis of the nine-banded armadillo (Dasypus novemcinctus)," J. Morphol. 233(3), 249-255 (1997).

11. D. Kelly, "Turtle and mammal penis designs are anatomically convergent," Proc. Biol. Sci. 271(Suppl. 5), S293-S295 (2004).

12. A. Balguid, M. P. Rubbens, A. Mol, R. A. Bank, A. J. Bogers, J. P. van Kats, B. A. de Mol, F. P. Baaijens, and C. V. Bouten, "The role of collagen cross-links in biomechanical behavior of human aortic heart valve leaflets-relevance for tissue engineering," Tissue Eng. 13(7), 1501-1511 (2007)

13. J. M. Gosline and R. E. Shadwick, "The mechanical properties of fin whale arteries are explained by novel connective tissue designs," $J$. Exp. Biol. 199(Pt 4), 985-997 (1996).

14. K. Fackler, L. Klein, and A. Hiltner, "Polarizing light microscopy of intestine and its relationship to mechanical behavior," J. Microsc. 124(Pt 3), 305-311 (1981).

15. P. Julkunen, P. Kiviranta, W. Wilson, J. S. Jurvelin, and R. K. Korhonen, "Characterization of articular cartilage by combining microscopic analysis with a fibril-reinforced finite-element model," J. Biomech. 40(8), 1862-1870 (2007).

16. L. Hughes, C. Archer, and I. ap Gwynn, "The ultrastructure of mouse articular cartilage: collagen orientation and implications for tissue functionality. A polarized light and scanning electron microscope study and review," Eur. Cells Mater 9, 68-84 (2005).

17. P. Kiviranta, J. Rieppo, R. K. Korhonen, P. Julkunen, J. Töyräs, and J. S. Jurvelin, "Collagen network primarily controls Poisson's ratio of bovine articular cartilage in compression," J. Orthop. Res. 24(4), 690-699 (2006).

18. K. Meek, "The cornea and sclera," Chapter 13 in P. Fratzl, Ed., Collagen: Structure and Mechanics, pp. 359-396, Springer, New York (2008).

19. C. Boote, S. Dennis, and K. Meek, "Spatial mapping of collagen fibril organization in primate cornea-an x-ray diffraction investigation," J. Struct. Biol. 146(3), 359-367 (2004).

20. T. T. Tower, M. R. Neidert, and R. T. Tranquillo, "Fiber alignment imaging during mechanical testing of soft tissues," Ann. Biomed. Eng. 30(10), 1221-1233 (2002).

21. P.-S. Jouk, A. Mourad, V. Milisic, G. Michalowicz, A. Raoult, D. Caillerie, and Y. Usson, "Analysis of the fiber architecture of the heart by quantitative polarized light microscopy. Accuracy, limitations and contribution to the study of the fiber architecture of the ventricles during fetal and neonatal life," Eur. J. Cardiothorac Surg. 31(5), 915-921 (2007).

22. W. C. Hutton, S. T. Yoon, W. A. Elmer, J. Li, H. Murakami, A. Minamide, and T. Akamaru, "Effect of tail suspension (or simulated weightlessness) on the lumbar intervertebral disc: study of proteoglycans and collagen," Spine 27(12), 1286-1290 (2002).

23. R. M. Korol, H. M. Finlay, M. J. Josseau, A. R. Lucas, and P. B. Canham, "Fluorescence spectroscopy and birefringence of molecular changes in maturing rat tail tendon," J. Biomed. Opt. 12(2), 024011 (2007).

24. Y. Xia, N. Ramakrishnan, and A. Bidthanapally, "The depth- dependent anisotropy of articular cartilage by Fourier-transform infrared imaging (FTIRI)," Osteoarthritis Cartilage 15(7), 780-788 (2007).

25. J. W. Jaronski and H. T. Kasprzak, "Linear birefringence measurements of the in vitro human cornea," Ophthalmic Physiol. Opt. 23(4), 361-369 (2003).

26. R. W. Knighton and X.-R. Huang, "Linear birefringence of the central human cornea," Invest. Ophthalmol. Visual Sci. 43(1), 82-86 (2002).

27. C. Pelletier, D. L. Keefe, and J. R. Trimarchi, "Noninvasive polarized light microscopy quantitatively distinguishes the multilaminar structure of the zona pellucida of living human eggs and embryos," Fertil. Steril. 81(Suppl 1), 850-856 (2004)

28. Y. Shen, T. Stalf, C. Mehnert, U. Eichenlaub-Ritter, and H.-R. Tinneberg, "High magnitude of light retardation by the zona pellucida is associated with conception cycles," Hum. Reprod. 20(6), 1596-1606 (2005).

29. L. Liu, J. R. Trimarchi, R. Oldenbourg, and D. L. Keefe, "Increased birefringence in the meiotic spindle provides a new marker for the onset of activation in living oocytes," Biol. Reprod. 63(1), 251-258 (2000).

30. P. Tran, E. Salmon, and R. Oldenbourg, "Quantifying single and bundled microtubules with the polarized light microscope," Biol. Bull. 189(2), 206 (1995).

31. P. T. Tran, S. Inoué, E. D. Salmon, and R. Oldenbourg, "Muscle fine structure and microtubule birefringence measured with a new polscope," Biophys. J. 187, 244-245 (1994).

32. H. Alhadlaq, Y. Xia, J. Moody, and J. Matyas, "Detecting structural changes in early experimental osteoarthritis of tibial cartilage by microscopic magnetic resonance imaging and polarised light microscopy," Ann. Rheum. Dis. 63(6), 709-717 (2004).

33. A. Benninghoff, "Form und Bau der Gelenkknorpel in ihren Beziehungen zur Funktion. Zweiter Teil: Der Aufbau des Gelenkknorpels in seinen Beziehungen zur Funktion (Form and structure of articular cartilage in their relation to function. Part two: the structure of articular cartilage in its relation to function)," Z. Zellforsch Mikrosk Anat 2. 783-862 (1925).

34. J. M. Clark, "Variation of collagen fiber alignment in a joint surface: a scanning electron microscope study of the tibial plateau in dog, rabbit, and man," J. Orthop. Res. 9(2), 246-257 (1991).

35. M. C. van Turnhout, M. B. Haazelager, M. A. Gijsen, H. Schipper, S. Kranenbarg, and J. L. van Leeuwen, "Quantitative description of collagen structure in the articular cartilage of the young and adult equine distal metacarpus," Animal Biol. 58(4), 353-370 (2008).

36. F. Massoumian, R. Juškaitis, M. Neil, and T. Wilson, "Quantitative polarized light microscopy," J. Microsc. 209(Pt 1), 13-22 (2003).

37. J. Rieppo, J. Hallikainen, J. S. Jurvelin, I. Kiviranta, H. J. Helminen, and M. M. Hyttinen, "Practical considerations in the use of polarized light microscopy in the analysis of the collagen network in articular cartilage," Microsc. Res. Tech. 71(4), 279-287 (2007).

38. S. Ross, R. Newton, Y.-M. Zhou, J. Haffegee, M.-W. Ho, J. Bolton, and D. Knight, "Quantitative image analysis of birefringent biological material," J. Microsc. 187(Pt 1), 62-67 (1997).

39. C. C. Hoyt and R. Oldenbourg, "Structural analysis with quantitative birefringence imaging," Am. Lab. (Shelton, Conn.) 31(14), 34-42 (1999).

40. R. Oldenbourg, "Analysis of microtubule dynamics by polarized light," Chapter 8 in J. Zhou, Ed., Microtubule Protocols, vol. 137 of Methods in Molecular Medicine, pp. 111-123, Humana Press, Totowa, NJ (2007).

41. J. P. Arokoski, M. M. Hyttinen, T. Lapveteläinen, P. Takács, B. Kosztáczky, L. Módis, V. Kovanen, and H. J. Helminen, "Decreased birefringence of the superficial zone collagen network in the canine knee (stifle) articular cartilage after long distance running training, detected by quantitative polarized light microscopy," Ann. Rheum. Dis. 55(4), 253-264 (1996).

42. K. Király, M. Hyttinen, T. Lapveteläinen, M. Elo, I. Kiviranta, J. Dobai, L. Módis, H. Helminen, and J. Arokoski, "Specimen preparation and quantification of collagen birefringence in unstained sections of articular cartilage using image analysis and polarizing light microscopy," Histochem. J. 29(4), 317-327 (1997).

43. H. E. Panula, M. M. Hyttinen, J. P. Arokoski, T. K. Långsjö, A. Pelttari, I. Kiviranta, and H. J. Helminen, "Articular cartilage superficial zone collagen birefringence reduced and cartilage thickness increased before surface fibrillation in experimental osteoarthritis," 
Ann. Rheum. Dis. 57(4), 237-245 (1998).

44. R. Appleyard, D. Burkhardt, P. Ghosh, R. Read, M. Cake, M. Swain, and G. Murrell, "Topographical analysis of the structural, biochemical, and dynamic biomechanical properties of cartilage in an ovine model of osteoarthritis," Osteoarthritis Cartilage 11(1), 65-77 (2003).

45. J. Rieppo, J. Hallikainen, J. Jurvelin, H. Helminen, and M. Hyttinen, "Novel quantitative polarization microscopic assessment of cartilage and bone collagen birefringence, orientation, and anisotropy," Trans. ORS 28, presented at the 49th Annual Meeting of the Orthopaedic Research Soceity, New Orleans, Paper 0570.

46. T. K. Långsjö, J. Rieppo, A. Pelttari, N. Oksala, V. Kovanen, and H. J. Helminen, "Collagenase-induced changes in articular cartilage as detected by electron-microscopic stereology, quantitative polarized light microscopy and biochemical assays," Cells Tissues Organs 172(4), 265-275 (2002).

47. R. Oldenbourg, "Polarization microscopy with the LC-microscope," Chapter 13 in R. D. Goldman and D. L. Spector, Eds., Live Cell Imaging: A Laboratory Manual, pp. 205-238, Cold Spring Harbor Laboratory Press, Woodbury, NY (2004).

48. D. J. Maitland and J. T. Walsh, Jr., "Quantitative measurements of linear birefringence during heating of native collagen," Lasers Surg. Med. 20(3), 310-318 (1997).

49. S. Kogure, H. Kohwa, and S. Tsukahara, "Effect of uncompensated corneal polarization on the detection of localized retinal nerve fiber layer defects," Ophthalmic Res. 40(2), 61-68 (2008).

50. X.-R. Huang and R. W. Knighton, "Microtubules contribute to the birefringence of the retinal nerve fiber layer," Invest. Ophthalmol. Visual Sci. 46(12), 4588-4593 (2005).

51. J. M. Bueno and J. Jaronski, "Spatially resolved polarization properties for in vitro corneas," Ophthalmic Physiol. Opt. 21(5), 384-392 (2001).

52. R. Oldenbourg and G. Mei, "New polarized light microscope with precision universal compensator," J. Microsc. 180(Pt 2), 140-147 (1995).

53. R. C. Jones, "A new calculus for the treatment of optical systems. I. Description and discussion of the calculus," J. Opt. Soc. Am. 31 488-493 (1941).

54. M. Shribak and R. Oldenbourg, "Techniques for fast and sensitive measurements of two-dimensional birefringence distributions," Appl. Opt. 42(16), 3009-3017 (2003).

55. E. Hecht, Optics, 2nd ed., Addison-Wesley, New York (1987).

56. E. Collett, Field Guide to Polarization, SPIE Press, Bellingham, WA
(2005).

57. M. Venn and A. Maroudas, "Chemical composition and swelling of normal and osteoarthrotic femoral head cartilage. I. Chemical composition," Ann. Rheum. Dis. 36(2), 121-129 (1977).

58. J. Rieppo, M. Hyttinen, E. Halmesmaki, H. Ruotsalainen, A. Vasara, I. Kiviranta, J. Jurvelin, and H. Helminen, "Changes in spatial collagen content and collagen network architecture in porcine articular cartilage during growth and maturation," Osteoarthritis Cartilage 17(4), 448-455 (2009).

59. R. Oldenbourg, E. Salmon, and P. Tran, "Birefringence of single and bundled microtubules," Biophys. J. 74(1), 645-654 (1998).

60. M. T. Nieminen et al., " $\mathrm{T}_{2}$ relaxation reveals spatial collagen architecture in articular cartilage: a comparative quantitative MRI and polarized light microscopic study," Magn. Reson. Med. 46(3), 487-493 (2001).

61. S. H. Bennett, "Methods applicable to the study of both fresh and fixed materials-the microscopical investigation of biological materials with polarized light," Chapter 9 in R. McClung Jones, Ed., McClung's Handbook of Microscopical Technique, 3rd ed., pp. 591-677, Cassel and Company, Hafner, NY (1950).

62. E. Saadat, H. Lan, S. Majumdar, D. M. Rempel, and K. B. King, "Long-term cyclical in vivo loading increases cartilage proteoglycan content in a spatially specific manner: an infrared microspectroscopic imaging and polarized light microscopy study," Arthritis Res. Ther 8(5), R147 (2006).

63. Y. Xia, J. B. Moody, H. Alhadlaq, and J. Hu, "Imaging the physical and morphological properties of a multi-zone young articular cartilage at microscopic resolution," J. Magn. Reson Imaging 17(3), 365374 (2003).

64. E. M. Slayter, Optical Methods in Biology, Wiley-Interscience, Huntington, NY (1970).

65. R. Teshima, T. Otsuka, N. Takasu, N. Yamagata, and K. Yamamoto, "Structure of the most superficial layer of articular cartilage," J. Bone Joint Surg. Br. 77(3), 460-464 (1995).

66. T. K. Långsjö, M. Hyttinen, A. Pelttari, K. Kiraly, J. Arokoski, and H. J. Helminen, "Electron microscopic stereological study of collagen fibrils in bovine articular cartilage: volume and surface densities are best obtained indirectly (from length densities and diameters) using isotropic uniform random sampling," J. Anat. 195(Pt 2), 281293 (1999).

67. R. Simon, "The connection between Mueller and Jones matrices of polarization optics," Opt. Commun. 42(5), 293-297 (1982). 